\title{
Stereo Matching Based On Election Campaign Algorithm
}

\author{
Qing Hua XIE ${ }^{1, a}$, Xiang Wei ZHANG ${ }^{1}$, Wen Ge LV ${ }^{1}$ and Si Yuan CHENG ${ }^{1}$ \\ ${ }^{1}$ School of Electromechanical Engineering, Guangdong University of Technology, Guangzhou china
}

\begin{abstract}
Stereo matching is one of the significant problems in the study of the computer vision. By getting the distance information through pixels, it is possible to reproduce a three-dimensional stereo. In this paper, the edges are the primitives for matching, the grey values of the edges and the magnitude and direction of the edge gradient were figured out as the properties of the edge feature points, according to the constraints for stereo matching, the energy function was built for finding the route minimizing by election campaign optimization algorithm during the process of stereo matching was applied to this problem the energy function. Experiment results show that this algorithm is more stable and it can get the matching result with better accuracy.
\end{abstract}

Keywords: Stereo Matching, Energy Function, Election Campaign Algorithm, Optimization.

\section{Introduction}

In binocular stereo vision, stereo matching get the image of the depth map from finding the scene with a point on the left and the right images.

According to the matching primitives, the matching to generate the disparity data distribution is different, the stereo matching can be based on region, feature, phase matching. According to constrains of the search strategy, stereo matching can be local and global matching. Noise, grey value difference, occlusion, perspective distortion will affect the match result. The method based on feature matching does not depend directly on the pixel gray, it has strong anti-interference ability, and fast calculation. Phase matching method lose effectiveness when the presence assumption of local structure does not exist. Local matching with low computational complexity, running fast, but is sensitive to occlusion and local ambiguity region. Global matching use the global optimal search strategy, which has better accuracy, can reduce the influence of occlusion and local ambiguity area such as texture consistency.

In this paper, the edge points are used as matching primitives, and the energy function with the constraint conditions of the stereo matching is established. In order to find the optimal solution path of energy function, election campaign algorithm is used to realize the optimal matching of feature points.

\section{Stereo Matching Disparity Map}

Edge points on the right and left two parallel binocular image were extracted, matching point set of each images is formed by the gray value and gradient. Matching energy function is established based on the constraint conditions, then election campaign algorithm is used to search the optimum, matched conjugate points and the parallax information.

\section{Get the Edge Points constraint conditions}

In this paper, Sobel Operator is used to extract edge points.

$$
\begin{aligned}
& S_{x}=-f(x-1, y-1)+f(x-1, y+1)-2 f(x, y-1)+2 f(x, y+1)-f(x+1, y-1)+f(x+1, y+1) \\
& S_{y}=f(x-1, y-1)+2 f(x-1, y)+f(x-1, y+1)-f(x+1, y-1)-2 f(x+1, y)-f(x+1, y+1)
\end{aligned}
$$

\footnotetext{
${ }^{a}$ Qing Hua XIE: xqhcool@163.com
} 
$f(x, y)$ is the grey value of point $(x, y)$, the gradient and direction angle at the point $(x, y)$ is defined as the formula below.

$$
\begin{gathered}
|\nabla f|=\left|S_{x}\right|+\left|S_{y}\right| \\
\theta(x, y)=\arctan \left|\frac{S_{y}}{S_{x}}\right|
\end{gathered}
$$

Energy function is defined as

$$
\begin{aligned}
& \nabla^{2} f=f(x-1, y-1)+f(x-1, y)+f(x-1, y+1)+f(x-1, y+1)+f(x, y-1)+ \\
& f(x, y+1)+f(x+1, y-1)+f(x+1, y)+f(x+1, y+1)-8+f(x, y)
\end{aligned}
$$

\section{Constraint Conditions}

Outer pole line constraint. In two images, a conjugate point in the corresponding outer pole line.

Uniqueness constraint. The point in the left image only has a conjugate point in the right image.

Consistency constraint. The matching feature points in the images should have similar properties.

Continuity constraint. Two points very close on the object, they should not have a big difference of depth.

Order preserving constraint. The sequence of two points in the left image will have same sequence of the corresponding matching points in the right image.

\section{Election Campaign Algorithm}

Election campaign algorithm (ECA) is a new optimization algorithm simulating election process. Election candidates always pursue the maximum support from voters by means of various election behaviors. They get the overview of their support from the voters according to the sample survey, and decide what to do on the next move. Solution space is imagined as voters and current solutions are imagined as candidates in ECA. The function value of a feasible solution is named as the prestige of a voter and the function value of a current solution is named as the prestige of a candidate.

In ECA, the candidates influence the voters round them, the voter's support is in proportion to the distance between the candidates and the voters. The voter will vote to the candidate which he pefer the most. The sum of location coordinates of every voters supported the candidate powered by its contribution is a new location coordinates, which is named support focus, it is the next position of the candidate. Such computational cycle is done continually until a candidate finds the position of the highest support, which is the global solution of the optimization problems.

Framework of the election campaign algorithm is described as the follows:

Set the Algorithm Parameters. Main parameters of Election campaign algorithm are:

- The number of candidates.

- The number of voters.

- The number of floating voters.

- Target accuracy.

Generate the Candidates and Calculate the Prestige of Them. Generated the define number of candidates in feasible solution field on the uniform distribution. Use the objective function to calculate the prestige of each candidate.

Generate the Voters. The uniform distribution is employed to generate the voters in feasible solution field.

Compute the Investigate Mean Square of Candidates. Higher prestige of a candidate, smaller the mean square deviation of local voters, so that ECA is able to converge to local optimization solution rapidly and steadily. The following formula is used to describe the relation of the prestige and the mean square deviation of a candidate.

$$
\sigma_{C_{i}}=\frac{\left(P_{M a x}-P_{\mathrm{C}_{i}}\right)}{\left(P_{M a x}-P_{M i n}\right)}
$$

Where $\sigma_{C_{i}}$ represents the mean square deviation of candidate $C_{i} ; P_{M a x}$ and $P_{M i n}$ are the maximum and minimum prestige of candidates.

Next step, voters are generated around each candidate on the normal distribution.

$$
N_{C_{i}}=\frac{\left(P_{\text {Max }}-P_{\mathrm{C}_{i}}\right)}{\left(P_{\text {Max }}-P_{\text {Min }}\right)}\left(N_{V}-N_{F V}\right)
$$


$N_{C_{i}}$ is the voter number of candidate $C_{i}$, $N_{V}$ is the number of all voters, $N_{F V}$ is the number of floating voters. Floating voters are generated on the uniform distribution. Here, it is supposed that candidate's effect on voters declined linearly.

Calculate the Supported of the Voters. Candidates can influence the voters within their effect range, the effect on voter $V_{j}$ from candidate $C_{i}$ is

$$
F_{C_{i} V_{j}}=\frac{D_{C_{i} M a x}-D_{C_{i} V_{j}}}{D_{C_{i} M a x}} P_{C_{i}}
$$

$F_{C_{i} V_{j}}$ is the effect on voter $V_{j}$ form candidate $C_{i}, V_{j}$ is the global or local voter. Here, it is supposed that candidate's effect on voters declined linearly.

Then Compute the prestige of voters by means of objective function.

The support of a voter is proportional to his prestige, and then the proportional constant will be reduced, so the prestige of a voter can used to denote the support of a voter directly. A voter may be influenced by several candidates; the voter should distribute his support to candidates proportionally on the magnitude of effect from candidate to voter. The support from voter $V_{j}$ to candidate $C_{i}$ is

$$
\begin{gathered}
F_{V_{j}}=\sum_{i=1}^{m} \max \left(F_{C_{1} V_{j}}, F_{C_{2} V_{j}}, \mathrm{~L}, F_{C_{i} V_{j}}, \mathrm{~L} F_{C_{m} V_{j}}\right)(9) \\
S_{C_{i} V_{j}}=\frac{F_{C_{i} V_{j}}}{F_{V_{j}}} P_{V_{j}}
\end{gathered}
$$

$S_{C_{i} V_{j}}$ represents the support from the voter $V_{j}$ to candidate $C_{i}$.

Find the Support Focus of Candidates. A new position coordinate will achieve by means of summing the products of the support from the voters to the candidate and the position coordinate of the voters. It is named the support focus.

$$
S_{C_{i}}=\sum_{j=1}^{n} \max \left(S_{C_{i} V_{1}}, S_{C_{i} V_{2}}, \mathrm{~L}, S_{C_{i} V_{j}}, \mathrm{~L} S_{C_{i} V_{n}}\right)
$$

$x_{C_{i}}^{*}=\frac{\sum_{j=1}^{n} S_{C_{i} V_{j}} x_{V_{j}}}{S_{C_{i}}}$

$x_{C_{i}}^{*}$ is the support focus of the candidate $C_{i}$. The support focus of a candidate is obtained by investigating, which depends on those voters whose distances to the candidate are nearer and the prestige is higher relatively. The next post of the candidate should be the support focus, where the candidate will have the higher support.

Calculate the Prestige of the Candidates and Compare the Prestige of the Voters with the Candidates. In order to jump out of local optimization solution and increase search rate, the prestige of candidates are compared to that of voters, if the prestige of a voter is higher than that of a candidate, the voter with higher prestige will substitute for the candidate and the that candidate of lower prestige will be eliminated in election.

Check Whether the Condition is Reach. Check whether the condition is reach, otherwise return to step B to execute the period. Here, the condition could be the number of select candidates in the target accuracy or max cycle. Do that circularly until the highest support is found.

$$
\left|P_{C_{i}}^{t}-P_{C_{i}}^{t-1}\right| \leq \xi
$$

$P_{C_{i}}^{t}$ represents the prestige of candidate $C_{i}$ in cycle t. When the absolute value of the difference between the prestige of the candidates in the present cycle and the previous cycle less than or equal to the target accuracy $\xi$, the computation stop.

Do that circularly until the highest support is found.

Flow scheme of the election campaign algorithm is shown in Fig. 1. 


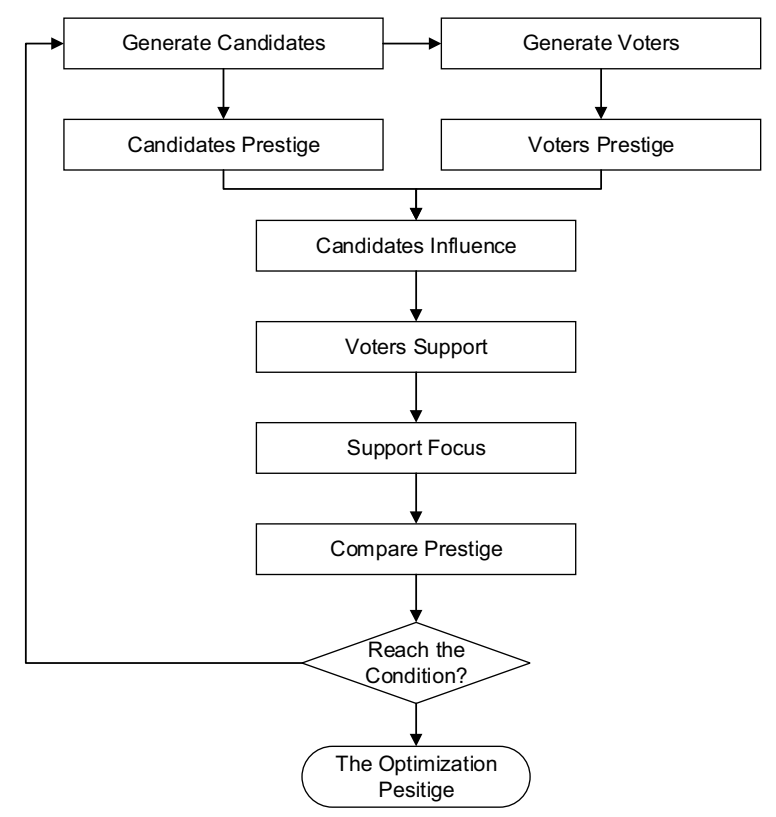

Fig. 1 Flow scheme of election campaign algorithm

\section{Stereo Matching Based On Election Campaign Algorithm}

The energy function is used as the object function of Election Campaign Algorithm. The matching relationship between edge feature point sets in the same corresponding line on the left and right images, $L\left\{l_{1}, l_{2}, \mathrm{~L}, l_{s}\right\}$ and $R\left\{r_{1}, r_{2}, \mathrm{~L}, r_{t}\right\}$, will find by the optimization algorithm.

Using Election campaign algorithm, the number of candidates define as 10 , the number of voters is 50 , the number of floating voters is 10 , and the target accuracy is set as $1 \%$. The experiment get the disparity map of two images, as is show in Fig. 2, it prove the feasibility of the algorithm.

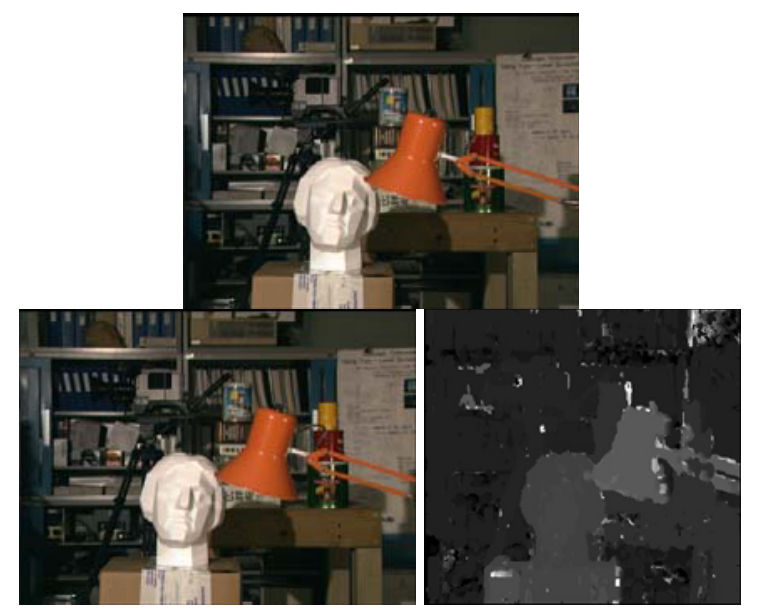

Fig. 2 Stereo matching experiment

\section{Conclusions}

With the analysis of the stereo matching algorithm and constraint conditions, this paper use edge feature points as matching primitives, and election campaign algorithm as the energy function optimal solution. The disparity map obtained from the results can show that algorithm is feasible.

Election campaign algorithm has good performance to avoid the solution trapped in local optima. This is particularly important when dealing with highly multimodal functions such as the matching of edge feature point sets. For future work, another feature and different energy function will be used in the matching of point sets.

\section{References}

[1] X. Su and T.M. Khoshgoftaar. A progressive edge-based stereo correspondence method. In Proc. of the Int. Symposium on Visual Compute. (ISVC), volume 1, pages 248 257, 2007.

[2] K. Kyung-Yoo, K. Sung-Yeol, and H. Yo-Sung. Stereo matching using global and local segmentation. In Proc. of the Int. Technical Conf. on Circus/Systems, Comp. and Communist. (ITC-CSCC 07), pages 1225 1226, 2007.

[3] X. Shenghua, L. Jiping, P. Jianping, and W. Yong. Straight line extraction for stereo image feature matching. In IEEE Proc. of the Congress on Image and Signal Processing, 2008 .

[4] G. Nieradka. Method for edge detection in images using fuzzy relation. In Proceedings of the SPIE, volume 6937, pages 69373E 69373E 8, 2008.

[5] Grzegorz Nieradka, Bohdan Butkiewicz, Features stereo matching based on fuzzy logic, IFSA-EUSFLAT 2009 Proceedings, 36, 1 (2009) 227-249.

Reference to a book: 
[6] SU Yin-jie, Stereo matching based on ant colony optimization algorithm, Micro computer information, 24, 8-3 (2008) 227-249. 